\title{
Zagreb Connection Number Index of Nanotubes and Regular Hexagonal Lattice
}

https://doi.org/10.1515/chem-2019-0007

received October 8, 2018; accepted November 8, 2018.

Abstract: Topological indices are the fixed numbers associated with the graphs. In recent years, mathematicians used indices to check the pharmacology characteristics and molecular behavior of medicines. In this article the first Zagreb connection number index is computed for the nanotubes $V C_{5} C_{7}[p, q], H C_{5} C_{7}[p, q]$ and Boron triangular Nanotubes. Also, the same index is computed for the Quadrilateral section $P_{m}^{n}$ and $P_{m+\frac{1}{2}}^{n}$
cuts from regular hexagonal lattices.

Keywords: Zagreb index; Modified first Zagreb connection number index; Molecular structure; Nanotubes.

\section{Introduction}

The molecular structure of every chemical drug can also be represented by a graph by considering the atoms as vertices of the graph and the bond between atoms as edges of the graph. The history and the mathematical concepts for graph theory are discussed in [1-3]. These days, computation of topological indices for different

*Corresponding author: Asfand Fahad, Department of Mathematics, COMSATS University Islamabad, Vehari Campus, Pakistan, E-mail: asfandfahad1@yahoo.com

Ansheng Ye: School of Geophysics, Chengdu University of Technology, Chengdu, 610059, China

Muhammad Imran Qureshi: Department of Mathematics, COMSATS University Islamabad, Vehari Campus, Pakistan

Adnan Aslam: Department of Natural Sciences and Humanities, University of Engineering and Technology, Lahore, Pakistan Muhammad Kamran Jamil: Department of Mathematics, Riphah Institute of Computing and Applied Sciences, Riphah International University, Lahore, Pakistann

Asim Zafar: Department of Mathematics, COMSATS University Islamabad, Vehari Campus, Pakistan

Rida Irfan: Department of Mathematics, COMSATS University

Islamabad, Sahiwal Campus, Pakistan chemical structures is an important task as discussed in [4-9].

If we denote a graph by $G(V(G), E(G))$, then $V(G)$ denoted the set of vertices of the graph $G$ and $E(G)$ denotes the set of edges of graph. The cardinality of $V(G)$ and $E(G)$ is called the order and size of the graph. The degree of vertex $v \in V(G)$ is the number of adjacent vertices to $v$ and is denoted by $d(v)$. The distance between the two vertices $u, v \in V(G)$ is denoted by $d(u, v)$ and is defined to be the length of the shortest path between $u$ and $v$.

A graph can be determined uniquely by the fixed numbers associated with it. Also, we can associate some numerical sequences with graphs. Similarly, some fixed numbers which are distance based and degree based can be associated with every graph. These invariants are called the topological indices.

Topological indices can also be associated with the molecular graphs of medicines. And with the help of these invariants, some pharmacology characteristics can be checked without using the laboratories and expensive materials.

Currently, many topological indices are being studied. The most studied among the degreed based topological indices are the first and second Zagreb indices. The first Zagreb index of graph $G$ is denoted and defined by

$$
M_{1}(G)=\sum_{v \in V(G)}(d(v))^{2}
$$

And the second Zagreb index of graph $G$ is denoted and defined by

$$
M_{2}(G)=\sum_{u v \in E(G)} d(u) d(v)
$$

Mathematical properties of first and second Zagreb indices are studied by Gutman el at. [10,11], Akhtar et al. [12] and Zhao et al. [13]. For further study of topological indices and related results see [14-21].

Throughout the paper, all the graphs under study are finite and connected. For a vertex $v \in V(G), \tau_{v}$ represents the number of vertices in the graph which are at the distance 2 from $v . \tau_{v}$ is called the connection number 
of $v$. By using the connection number, a topological index named "the Modified first Zagreb connection index" is defined as

$$
Z C_{1}^{*}(G)=\sum_{v \in V(G)} d(v) \tau_{v}
$$

$Z C_{1}^{*}$ was first introduced by Ali et al. [22]. Wang et al. [23], computed $Z C_{1}^{*}$ for different molecular structures of dendrimers.

\section{Motivation}

According to the International Academy of Mathematical Chemistry, in order to identify whether any topological index is useful for prediction of chemical properties, the correlation between the values of that topological index for different octane isomers and parameter values related to certain physicochemical property of them should be considered. Generally octane isomers are convenient for such studies, because the number of the structural isomers of octane is large [24] enough to make the statistical conclusion reliable. Ali et al [22] checked the correlation ability of $Z C_{1}^{*}$ for the following thirteen physicochemical properties of octane isomers: boiling point, density, heat capacity at $\mathrm{P}$ constant, entropy, heat capacity at $\mathrm{T}$ constant, enthalpy of vaporization, acentric factor, standard enthalpy of vaporization, enthalpy of formation, octanol-water partition coefficient, standard enthalpy of formation, total surface area and molar volume. They concluded that $Z C_{1}^{*}$ yields the correlation coefficient, which is approximately 0.892 and 0.949 for entropy and acentric factor, respectively. This observation suggests that the molecular descriptor $Z C_{1}^{*}$ may be helpful in quantitative structure-property relationship and quantitative structure-activity relationship studies, and hence this descriptor may be considered for further investigations.

\section{Zagreb Connection Number Index for Nano-tubes}

Since the discovery of $C_{60}$, carbon nanotubes as well as graphenes, the precursor of carbon fullerenes and carbon nanotubes have attracted wide attention due to their electronic properties and great potential applications[25,26]. There is a growing interest in exploring the structure and energetic of these pure carbon

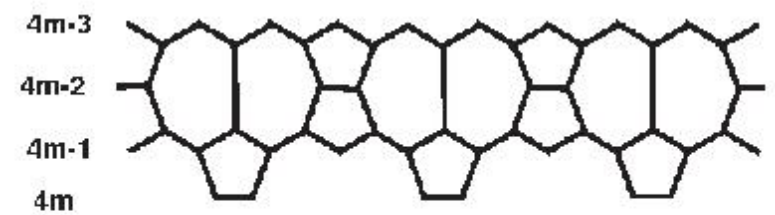

$m$-th period of $V C_{5} C_{7}$ nanotube.

Figure 1:

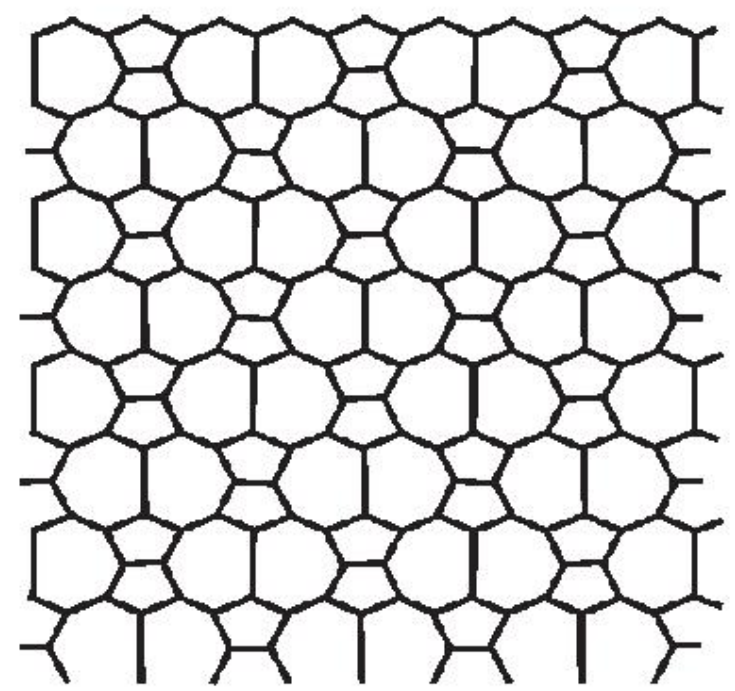

The graph of $V C_{5} C_{7}[p, q]$ nanotube with $p=3$ and $q=4$.

Figure 2:

clusters, carbon nanotubes with width on the nanometer scale, and carbon containing molecules because they are expected to have wide applications.

There are various types of nano-tubes which are under study. Some topological indices are found for nano-tubes [24]. The nano-tube $V C_{5} C_{7}[p, q],(p, q>1)$ is a net which is constructed by altering the $C_{5}$ and $C_{7}$ following the trivalent decoration. Using this type of tiling, we can cover a cylinder or a torus. Here $p$ is a number of pentagons and $q$ represents the number of periods. $q$ is the number of periods in lattice.

In Figure 1, one period of $V C_{5} C_{7}$ is shown. In one period there are 4 rows. In one period there are $16 p$ vertices. And there are $3 p$ vertices which are joined at the other end of the graph. Hence $\left|V\left(V C_{5} C_{7}[p, q]\right)\right|=16 p q+3 p$. In Figure 2 the graph of $V C_{5} C_{7}[p, q],(p, q>1)$ is shown when $p=3, q=4$.

Theorem3.1:Let $G=V C_{5} C_{7}[p, q]$, where $p, q>1$. 
Then

$$
Z C_{1}^{*}(G)=288 p q-60 p
$$

Proof: For $G=V C_{5} C_{7}[p, q]$, out of total $16 p q+3 p$ vertices, $3 p$ vertices have degree $2,3 p$ have degree 1 and the remaining $16 p q-3 p$ have degree 3 . The connection number for the different vertices is given in next table

From the Table 1 and definition of modified first Zagreb connection index we have

$$
\begin{gathered}
Z C_{1}^{*}(G)=\sum_{v \in V(G)} d(v) \tau_{v} \\
=2 \times 4 \times 3 p+1 \times 2 \times 3 p+3 \times 4 \times 6 p+3 \times 6 \times(16 p q-9 p)
\end{gathered}
$$

After simplification

$$
Z C_{1}^{*}(G)=288 p q-60 p
$$

The nano-tube $H C_{5} C_{7}[p, q],(p, q>1)$ is a net of two dimensional lattice which is constructed by altering the $C_{5}$ and $C_{7}$ following the trivalent decoration. Using this type of tiling, we can cover a cylinder or a torus. Here $p$ is a number of Heptagons and $q$ represents the number of periods in lattice. In 2-dimensional lattice of $H_{5} C_{7}[p, q]$, $p$ is the number of heptagons in one period.

In Figure 3, one period of $\mathrm{HC}_{5} \mathrm{C}_{7}$ is shown for better understanding. One period consisting of 4 rows and $16 p$ vertices. $2 p$ vertices are joined at the other end of the graph. Hence, $\left|V\left(H C_{5} C_{7}[p, q]\right)\right|=16 p q+2 p$. In Figure 4, $H C_{5} C_{7}[p, q],(p, q>1)$ is given for $p=q=3$.

Theorem 3.2: Let $G=H C_{5} C_{7}[p, q],(p, q>1)$. Then

$$
Z C_{1}^{*}\left(H C_{5} C_{7}[p, q]\right)=288 p q-46 p
$$

Proof: For $\mathrm{HC}_{5} \mathrm{C}_{7}[p, q]$, out of total number of vertices $16 p q+2 p$, the $2 p$ vertices have degree $1,2 p$ vertices have degree 2 and the remaining $16 p q-2 p$ have degree 3. The connection number for different vertices is given in next table

Now by the Table 2 and definition of the modified first Zagreb connection index we have

$$
\begin{aligned}
& Z C_{1}^{*}(G)=\sum_{v \in V(G)} d(v) \tau_{v} \\
& =1 \times 2 \times 2 p+2 \times 4 \times 2 p+3 \times 4 \times 5 p+3 \times 6 \times(16 p q-7 p)
\end{aligned}
$$

After simplification we have

$$
Z C_{1}^{*}\left(H C_{5} C_{7}[p, q]\right)=288 p q-46 p
$$

Table 1:

$$
\begin{array}{ccc}
d(v) & \tau_{v} & \text { no. of vertices } \\
2 & 4 & 3 p \\
1 & 2 & 3 p \\
3 & 4 & 6 p \\
3 & 6 & 16 p q-9 p
\end{array}
$$

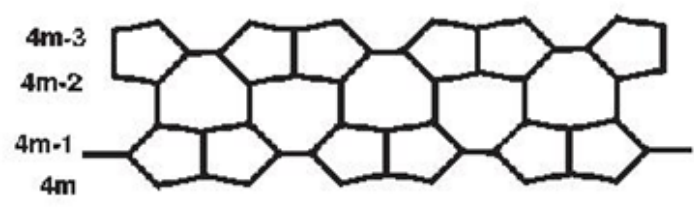

$m$-th period of $\mathrm{HC}_{5} \mathrm{C}_{7}$ nanotube.

Figure 3:

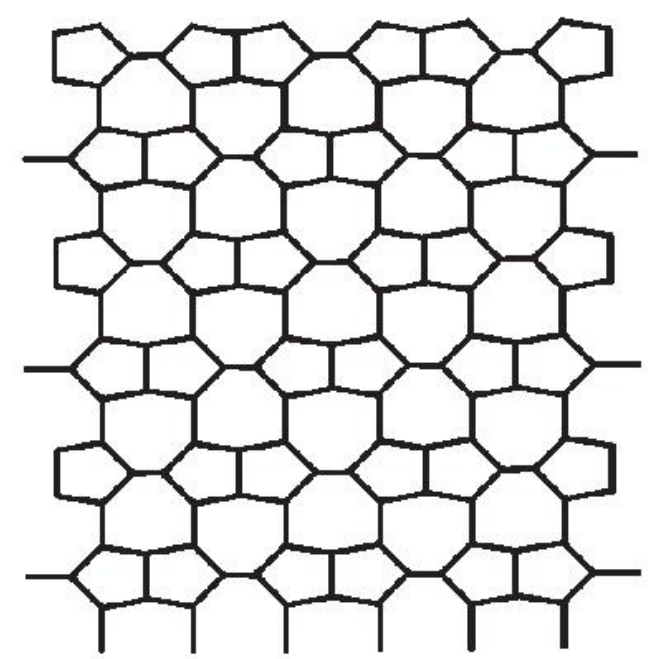

The graph of $H C_{5} C_{7}[p, q]$ nanotube with $p=3$ and $q=3$.

Figure 4:

Table 2:

$\begin{array}{ccc}d(v) & \tau_{v} & \text { No of vertices } \\ 1 & 2 & 2 p \\ 2 & 4 & 2 p \\ 3 & 4 & 5 p \\ 3 & 6 & 16 p q-7 p\end{array}$




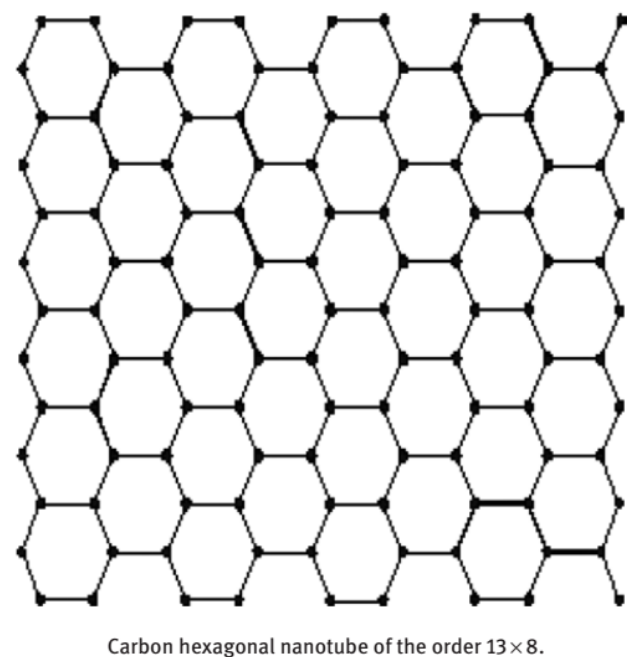

Figure 5:

The Boron Nanotube was originally predicted by Boustani and Quandt et al. [27]. It was proposed that the most suitable structure of $C_{20}$ is a double ring tabular structure, which can be considered as the embryo of single walled Boron Nanotubes. In 2004, Ciuparu et al. [28] successfully synthesized pure Boron single walled nanotubular structures with the diameter in the range of $3 \mathrm{~nm}$ and thus confirmed the existence of Boron Nanotubes.

A carbon hexagonal Nanotube of order $m \times n$ is a tube obtained from a carbon hexagonal sheet of order $m \times n$ by merging the vertices of last column with respective vertices of first column. (see Figure 5)

A Boron triangular Nanotube of order $m \times n$ is obtained from the hexagonal nanotubes of order $m \times n$ by adding a new vertex in the center of each hexagon and connecting it to all vertices of the hexagon (see Figure 6).

We denote the Boron nanotube of order $m \times n$ by $B T[m, n]$. Boron nanotubes have an odd number of rows and an even number of columns. This fact can be observed from the following theorem.

Theorem [29]:

A Boron triangular nanotube of order $m \times n$ has $\frac{3 m n}{2}$ vertices and $\frac{3 n(3 m-2)}{2}$ edges.

In the next theorem, we will calculate the Zagreb connection index of Boron triangular nanotube.

Theorem3.3: Let $G$ be the graph of Boron nanotube with $m, n \geq 2$.. Then the Zagreb connection index is

$$
Z C_{1}^{*}(G)=6 m(18 n-31)
$$

Proof: It can be observed from the molecular graph of Boron nanotubes that the $3 m$ vertices of the first

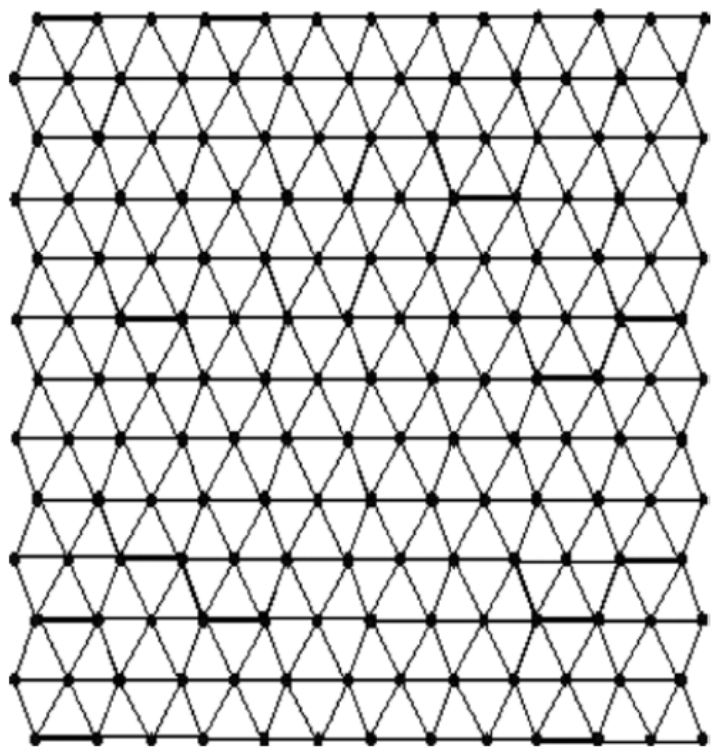

Boron triangular nanotube of the order $13 \times 8$.

Figure 6:

row and last row are of degree 4, while the remaining $\frac{3 m n}{2}-3 m$ vertices are of degree 6 . All the top and bottom row vertices have connection number 7 , whereas, among the remaining vertices $3 m$ have connection number 9 and $\frac{3 m n}{2}-6 m$ have connection number 12 . Now using the formula, we can calculate the Zagreb connection index as

$$
Z C_{1}^{*}=\sum_{v \in V(G)} d(v) \tau_{v}
$$

$$
\begin{gathered}
Z C_{1}^{*}(G)=4 \times 7 \times 3 m+6 \times 9 \times 3 m+6 \times 12\left(\frac{3 m n}{2}-3 m\right) \\
=6 m(18 n-31)
\end{gathered}
$$

\section{Zagreb Connection number index for regular Hexagonal Lattice}

There are various types of lattices which are under study. Some topological indices are obtained for hexagonal lattices [30].

If $L$ is a regular hexagonal lattice, let $P_{m}^{n}$ be $m \times n$ quadrilateral section cut from $L$ where $m, n \geq 2$. Here $m$ represents the number of hexagons on the top and bottom sides and $n$ is the number of hexagons on the lateral sides. If we identify the two lateral sides of $P_{m}^{n}$, we will obtain the cylinder. Further, if we identify the top and bottom sides by identifying $u_{1}^{0}$ to $u_{1}^{n}$ and $v_{1}^{0}$ to $v_{1}^{n}$, 


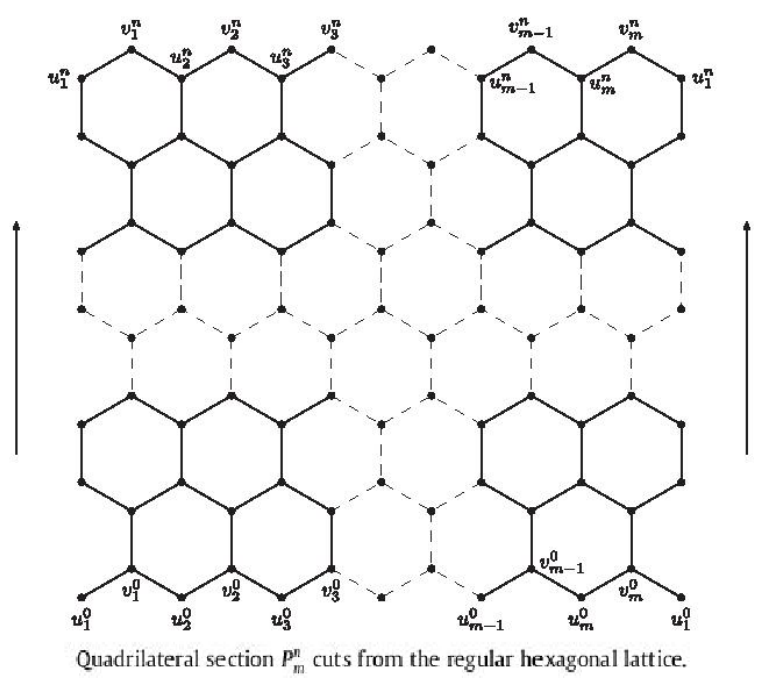

Figure 7:

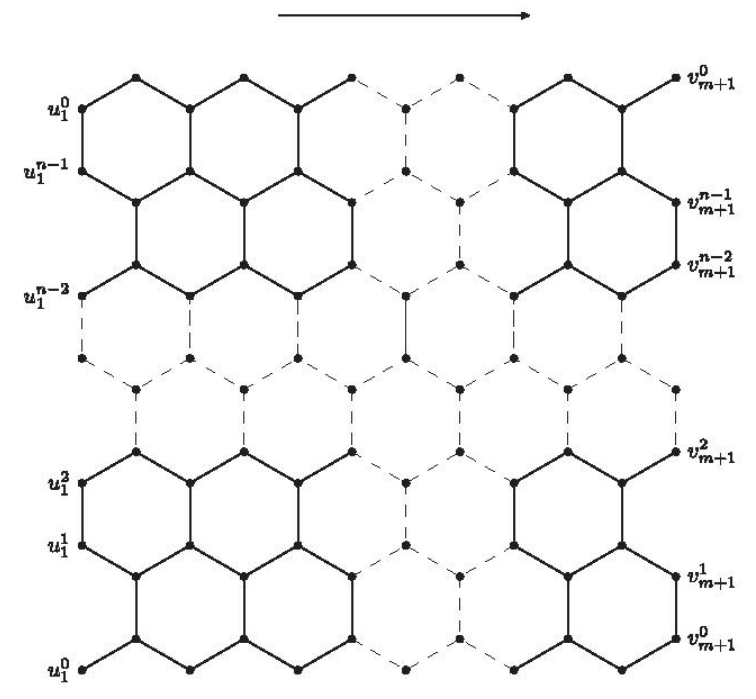

Quadrilateral section $P_{m+1 / 2}^{n}$ cuts from the regular hexagonal lattice.

Figure 8:

$\forall i=1,2, \ldots, m$, we obtain the toroidal fullerence with $m n$ hexagons. Shown in Figure 7.

If $V\left(P_{m}^{n}\right)$ is the set of vertices then $\left|V\left(P_{m}^{n}\right)\right|=2 m n$.

Theorem 4.1: If $G=P_{m}^{n} \quad$ where $\quad m, n \geq 2$., then

$$
Z C_{1}^{*}(G)=36 m n
$$

Proof: Since the total vertices are $2 m n$. From the structure it is clear that the degree of all vertices is 3 . The connection number for all the vertices is 6 . Hence, by definition of the modified first Zagreb connection index

$$
\begin{gathered}
Z C_{1}^{*}=\sum_{v \in V(G)} d(v) \tau_{v} \\
Z C_{1}^{*}\left(P_{m}^{n}\right)=3 \times 6 \times 2 m n
\end{gathered}
$$

Let $L$ be a regular hexagonal lattice. Let $P_{m+1 / 2}^{n}$ be a quadrilateral section cut from $L$.

Where $m \geq 1, n \geq 2$. Here $m+1 / 2$ is the number of hexagons on the top and bottom sides and $n$ represents the no of hexagons on the lateral sides. If we identify the top and bottom sides of $P_{m+1 / 2}^{n}$, a cylinder will be obtained. After that if we identify the lateral sides of cylinder such that, identify $u_{1}^{0}$ to $v_{m+1}^{0}$ and $u_{1}^{j}$ to $v_{m+1}^{n-j}$, $\forall j=1,2, \ldots, n-1$ to obtain the Klien bottle. Shown in Figure 8. If $V\left(P_{m+1 / 2}^{n}\right)$ is the set of vertices then $\left|V\left(P_{m+1 / 2}^{n}\right)\right|=2 n(m+1 / 2)$.

Theorem 4.2: Let $G=P_{m+1 / 2}^{n}$ where $\mathrm{m}>1, \mathrm{n}>2$, then

$$
Z C_{1}^{*}(G)=36 n\left(m+\frac{1}{2}\right)
$$

Proof: Since the total vertices are $2 n(m+1 / 2)$. From the structure it is clear that the degree of all vertices is 3 . The connection number for all the vertices is 6 . Hence by definition of the modified first Zagreb connection index

$$
\begin{gathered}
Z C_{1}^{*}=\sum_{v \in V(G)} d(v) \tau_{v} \\
Z C_{1}^{*}\left(P_{m+1 / 2}^{n}\right)=3 \times 6 \times 2 n\left(m+\frac{1}{2}\right) \\
Z C_{1}^{*}\left(P_{m+1 / 2}^{n}\right)=36 n\left(m+\frac{1}{2}\right)
\end{gathered}
$$

\section{Conclusion}

Topological indices of molecular graphs are helpful to study the properties of drugs and manufacture the medicines. Therefore, they have been widely studied [1-3, 5, 6, 10-15, 29-31]. The main aim of this paper is to study the modified first Zagreb index of some famous nanotubes and quadrilateral section $P_{m}^{n}$ and $P_{m+\frac{1}{2}}^{n}$ cuts from the regular hexagonal lattice, which appears very frequently in the literature. As modified, the first Zagreb index can been used in QSPR/QSAR study and can play a crucial role in analyzing both the entropy and acentric factor for chemical compounds. The results obtained in our paper illustrate the promising prospects of application for chemical and nanosciences. For future direction, we want 
to remark that the same technique can be used to compute the modified first Zagreb index for different networks.

Acknowledgement: This work is supported by National Key Research and Development Program under Grant 2016YFB0800600.

The authors (Muhammad Imran Qureshi and Asfand Fahad) gratefully acknowledge ORIC, COMSATS University Islamabad, Pakistan for supporting this research under the grant of project number 16-52/CRGP/CIIT/VEH/17/1141.

Ethical approval: The conducted research is not related to either human or animal use.

Conflict of interest: Authors declare no conflict of interest.

\section{Reference}

[1] Gutman I., Polansky O. E., Mathematical Concepts in organic chemistry, Berlin: Springer, 1986.

[2] Goodman J. E., Orourke J., Handbook of Discrete and Computational Geometry, CRC Press LLC, 1997, 225-242.

[3] Trinajstic N., Chemical graph theory, 2nd revised ed., Florida: CRC Press, Boca Raton, 1993.

[4] Furtula B., Gutman I., A forgotten topological index, J. Math. Chem., 2015, 53(4), 1184-1190.

[5] Goubko M., Minimizing degree-based topological indices for trees with given number of pendent vertices, MATCH Commun. Math. Comput. Chem, 2014, 71, 33-46.

[6] Munir M., Nazeer W., Kang S. M., Qureshi M. I., Nizami A. R., Kwun Y. C., Some Invariants of Jahangir Graphs, Symmetry, 2017, 9(17), 1-15.

[7] Gao W., Siddiqui M. K., Imran M., Jamil M. K., Farahani M. R., Forgotten topological index of chemical structure in drugs, Saudi Pharm. Journal, 2017, 25, 280-286.

[8] Bashir Y., Aslam A., Kamran M., Qureshi M. I., Jahangir A., Rafiq M., et al., On Forgotten Topological Indices of Some Dendrimers Structure, Molecules, 2017, 22(867), 1-8.

[9] Shao Z., Wu P., Zhang X., Dimitrov D., Liu J., On the maximum $A B C$ index of graphs with prescribed size and without pendent vertices, IEEE Access, 2018, 6, 27604--27616.

[10] Gutman I., Das K. C., The first zagreb index 30 years after, MATCH Commun. Math. Comput. Chem., 2004, 50, 83-92.

[11] Gutman I., Jamil M. K., Akhtar N., Graph with fixed number of pendent vertices and minimal first zagreb index, Transactions on Combinatorics, 2015, 4(1).

[12] Akhtar N., Jamil M. K., Tomescu I., External first and second Zagreb indices of apex trees, U. P. B. Sci. Bull. Series A, 2016, 78(4), 221-230.

[13] Zhao Q., Li S., Sharp biunds for the Zagreb indices of bicyclic graphs with k-pendent vertices, Discrete Appl. Math., 2010, 158, 1953-1962.
[14] Aslam A., Jamil M. K., Gao W., Nazeer W., Topological Aspects of Some dendrimer structures, Nanotechnology Reviews, 2018, 7(2), 123-128.

[15] Aslam A., Ahmad S., Gao W., On Certain Topological Indices of Boron Triangular Nanotubes, Z. Naturforsch, 2017, 72(8), 711716.

[16] Aslam A., Bashir Y., Ahmad S., Gao W., On Topological Indices of Certain Dendrimer Structures, Z. Naturforsch, 2017, 72(6), 559-566.

[17] Sardar M. S., Zafar S., Farahani M. R., The Generalized Zagreb Index of Capra-Designed Planar Benzenoid Series C a k, Open J. Math. Sci.,2017, 1(1), 44-51.

[18] Noreen S., Mahmood A., Zagreb Polynomials and Redefined Zagreb Indices for the line graph of Carbon Nanocones, Open J. Math. Anal., 2018, 2(1), 67-76.

[19] Gao W., Asif M., Nazeer W., The Study of Honey Comb Derived Network via Topological Indices. Open J. Math. Anal., 2018, 2(2), 10-26.

[20] Aslam A., Guirao J. L. G., Ahmad S., Gao W., Topological indices of line graph of subdivision graph of complete bipartite graphs, Applied Mathematics and Information sciences, 2017, 11(6), 1631-1636.

[21] De N., Hyper Zagreb Index of Bridge and Chain Grpahs, Open J. Math. Sci., 2018, 2(1), 1-17.

[22] Ali A., Trinsjstic N., A novel/old modification of the first Zagreb index, arXiv:1705-10430v1.

[23] Wang X. L., Liu J. B., Jamil M. K., Qureshi M. I., Fahad A., Farahani M. R., Zagreb Connection index of Drugs related chaemical structures, Turkish Journal of Medical Sciences (In Press).

[24] Hayat S., Imran M., Computation of Certain Topological Indices of Nanotubes Covered by $\mathrm{C} 5$ and C7, J. Computational and Theoretical Nanoscience, 2015, 12, 1-9.

[25] Lijima S., Helical Microtubules of Graphitic Carbon, Nature, 1991, 354, 56-58.

[26] Baughman R. H., Zakhidov A. A., De Heer W. A., Carbon Nanotubes--the Route toward Applications, Science, 2002, 297(5582), 787-792.

[27] Boustani I., Quandt A., Hernandez E., Rubio A., New boron based nanostructured materials, The Journal of Chemical Physics, 1999, 110(3176).

[28] Ciuparu D., Klie R. F., Zhu Y., Pfefferle L., Synthesis of Pure Boron Single-Wall Nanotubes, The Journal of Physical Chemistry, 2004, 108(13), 3967-3969.

[29] Manuel P., Computational Aspects of Carbon and Boron Nanotubes, Molecules, 2010, 15(12), 8709-8722.

[30] Baca M., Horvathova J., Mokrisova M., Suhanyiova A., On topological indices of fullerenes, Applied Mathematics and Computation, 2015, 251, 154-161.

[31] Shao Z., Wu P., Gao Y., Gutman I., Zhang X., On the maximum ABC index of graphs without pendent vertices, Applied Mathematics and computation, 2017, 315, 298-312. 